\title{
ANALYSIS OF HIGH-RESOLUTION MICROELECTRODE EEG RECORDINGS IN AN ANIMAL MODEL OF SPONTANEOUS LIMBIC SEIZURES
}

\author{
C. KOMALAPRIYA*,†, , M. C. ROMANO ${ }^{\S}$, M. THIEL ${ }^{\S}$, \\ U. SCHWARZ* and J. KURTHS ${ }^{*, \dagger,}$, \\ ${ }^{*}$ Centre for Dynamics of Complex Systems, University of Potsdam, \\ 14476 Potsdam, Germany \\ ${ }^{\dagger}$ Potsdom Institute for Climate Impact Research, \\ 14412 Potsdam, Germany \\ ${ }^{\ddagger}$ komala@agnld.uni-potsdam.de \\ $\S$ Department of Physics, University of Aberdeen, \\ Aberdeen, $A B 24$ 3UE, $U K$ \\ \Department of Physics, Humboldt University, Berlin, \\ 12489 Berlin, Germany \\ J. SIMONOTTO \\ School of Computing Science, University of Newcastle upon Tyne, \\ NE1 $7 R U, U K$ \\ M. FURMAN \\ Department of Engineering, University of Cambridge, \\ Cambridge, CB2 1PZ, UK \\ W. L. DITTO and P. R. CARNEY \\ Department of Biomedical Engineering, University of Florida, \\ FL 32611, USA
}

Received April 14, 2008; Revised May 6, 2008

\begin{abstract}
We perform a systematic data analysis on high resolution $(0.5-12 \mathrm{kHz})$ multiarray microelectrode recordings from an animal model of spontaneous limbic epilepsy, to investigate the role of high frequency oscillations and the occurrence of early precursors for seizures. Results of spectral analysis confirm the importance of very high frequency oscillations (even greater than $600 \mathrm{~Hz}$ ) in normal (healthy) and abnormal (epileptic) hippocampus. Furthermore, we show that the measures of Recurrence Quantification Analysis (RQA) and Recurrence Time Statistics (RTS) are successful in indicating, rather uniquely, the onset of ictal state and the occurrence of some warnings/precursors during the pre-ictal state, in contrast to the linear measures investigated.
\end{abstract}

Keywords: Multiarray microelectrode; EEG recordings; RQA; RTS. 


\section{Introduction}

Epilepsy is one of the most common pathological neural disorder characterized by sudden and recurrent malfunctions of the brain, called seizures. Sudden incidence of seizures can be dangerous and life threatening. Hence, the prediction/anticipation of epileptic seizures is a topic of great interest, as it would help to improve therapeutic methods through the development of warning devices. A wide range of linear and nonlinear measures [Mormann et al., 2005; Iasemidis et al. 2005; Worrell et al., 2004; Litt et al., 2001; Jerger et al., 2001; Mormann et al., 2003; Quyen et al., 1999; Lehnertz \& Elger, 1995, 1999; Casdagli et al., 1997; Steuer et al., 2004; Li et al., 2004; Thomasson et al., 2001] have been suggested for the prediction of seizures. But currently there exists no widely accepted algorithm for characterizing the transitions of the brain from a normal to the pre-ictal and then to the ictal (seizure) state, based on macroscopic EEG (Electroencephalogram) recordings, due to many reasons [Mormann et al., 2007]. Since the form and the type of seizure vary greatly between individuals, devising a robust algorithm for seizure anticipation is a complicated task. One possibility to tackle this problem is to identify the basic mechanisms governing brain dynamics that lead to the occurrence of a seizure [Sanchez et al., 2006]. However, the knowledge about these main mechanisms is still very limited.

The ongoing progress in the neural interface technology has put forth another interesting question: what spectrum of oscillations within EEG are of significance for physiological and diagnostic/therapeutic applications? Recent developments in multielectrode technology has enabled us to access and analyze oscillations at the higher end of the frequency spectrum. Some early studies of high frequency oscillations $(80-500 \mathrm{~Hz})$ from nonprimates and humans already indicate the significance of the high frequency oscillations in memory consolidation [Buzsaki, 1989; Buzsaki, 1996; Buzsaki et al., 1992; Buzsaki, 1998; Clemens et al., 2007] and seizure generation [Bragin et al., 1999a; Bragin et al., 1999b; Bragin et al., 2002a; Bragin et al., 2002b; Bragin et al., 2004]. Few fresh studies also indicate the importance of further higher frequency oscillations $(>600 \mathrm{~Hz})$ in some physiological activities and epileptogenesis [Sanchez et al., 2006; Talathi et al., 2008; Worrell et al., 2008; Firpi et al., 2007].
In this paper, we investigate high resolution intracranial EEG recordings $(0.5-12 \mathrm{kHz})$ obtained in vivo from a rat which has been induced to spontaneously seize (see Sec. 2). We analyze the data by applying linear methods, such as moments, spectral analysis and correlation function, and also nonlinear methods based on the concept of recurrence [Marwan et al., 2007]. Until now, recurrence based analysis of EEG data has concentrated in capturing the nonstationarity of the ictal stage (loss of recurrence) [Li et al., 2004; Thomasson et al., 2001]. However, here, we adopt a different strategy to detect dynamical changes in the data.

The main aim of our study is to analyze the very highly sampled microarray electrodes recordings so as to understand the importance of higher frequency oscillations in seizure analysis and their dynamics. Unlike in traditional epilepsy research, where control data is also obtained from subjects who are suffering from epilepsy, we investigate the control data that is obtained from healthy subjects. This approach will enable us to compare the role of higher frequency oscillations in healthy and diseased states. The central question is of course to look for unique warnings/ precursors occurring before a seizure (the pre-ictal stage) which would help in an early seizure anticipation. Furthermore, we compare the results from linear measures with that of recurrence based measures (RQA and RTS).

The organization of this paper is as follows. In Sec. 2, we present the details of the data that we investigate. Next, in Sec. 3 we describe briefly the measures we employ to analyze the EEG data. In Sec. 4, we present and compare the results obtained from various methods and finally, we conclude in Sec. 5 .

\section{Data Description}

The EEG data comes from the rat model of chronic limbic epilepsy using a micro-electrode array, implanted in the ipsi-/contra- hippocampal, the CA3-CA1 and the dentate gyrus regions. The animal model of chronic limbic epilepsy is a well documented model in literature and is associated with recurrent spontaneous hippocampal seizures. Micro-electrode array were surgically implanted on the above specified sites of rats brain based on earlier studies of their role in the generation of epilepsy. Self-sustained limbic status epilepticus was induced by hippocampal stimulation after one week of surgery. Immediately 
after the stimulation and later the animal was found to seize and the seizure detection was performed by a group of experts monitoring both video and EEG data. A detailed description about model preparation, micro-electrode array specifications/placement/implantation surgery and induction of status epilepticus can be found in [Sanchez et al., 2006; Talathi et al., 2008]. The multichannel EEG potentials were recorded, synchronously, with the sampling rate $24414.1 \mathrm{~Hz}$, which corresponds to a sampling time of $\Delta t=41 \mu \mathrm{s}$. The data was band pass filtered between $0.5-12000 \mathrm{~Hz}$ before recording and the analog to digital conversion was made with 16-bit analog to digital convertor.

Specifically, in this paper, we analyze three segments of 30 min intracranial EEG recordings taken from eight channels implanted in the hippocampus subfields CA1, CA3 and dentate gyrus. Each of the 30 min segments were a part of continuous synchronous multichannel recordings collected over 73 days. Microelectrodes $1,2,7$ and 8 are located in the CA1 and CA3 regions, and microelectrodes 3-6 are in the dentate gyrus (Fig. 1). The stimulation electrode was implanted in the contralateral posterior ventral hippocampus [Sanchez et al., 2006; Talathi et al., 2008].

One of the three segments corresponds to the quiet wakeful normal state of a healthy rat (where the animal was either sleeping or quietly exploring its cage) before the hippocampal stimulation - control data. The remaining two data sets correspond to quite wakefullness after the stimulation during which seizure-1 and the seizure-2 were observed. The control EEG data [Fig. 2(a)] that we analyzed was found to contain some artifacts like those at around $10 \mathrm{~s}, 90 \mathrm{~s}, 155 \mathrm{~s}$ and 175 s. Furthermore, the control data showed a continuous high frequency, seizure activity between 100-160 s and some instantaneous high frequency bursts at some later instances as well. The onset of the ictal phase in the seizure- 1 segment is at about $1150 \mathrm{~s}$ and that of the seizure- 2 segment is at

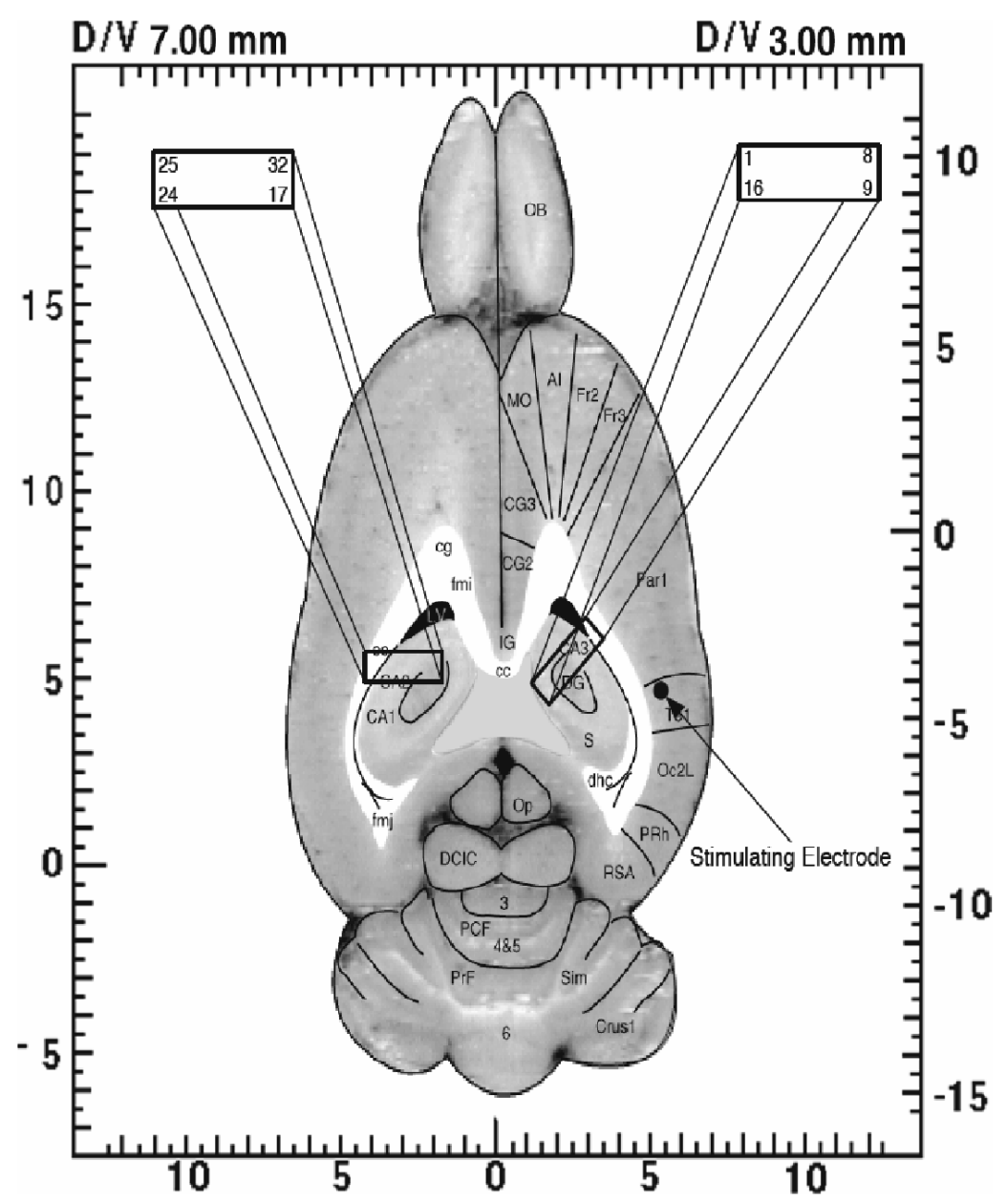

Fig. 1. A horizontal section of the rat's brain showing the schematic arrangement of the electrodes [Talathi et al., 2008]. 


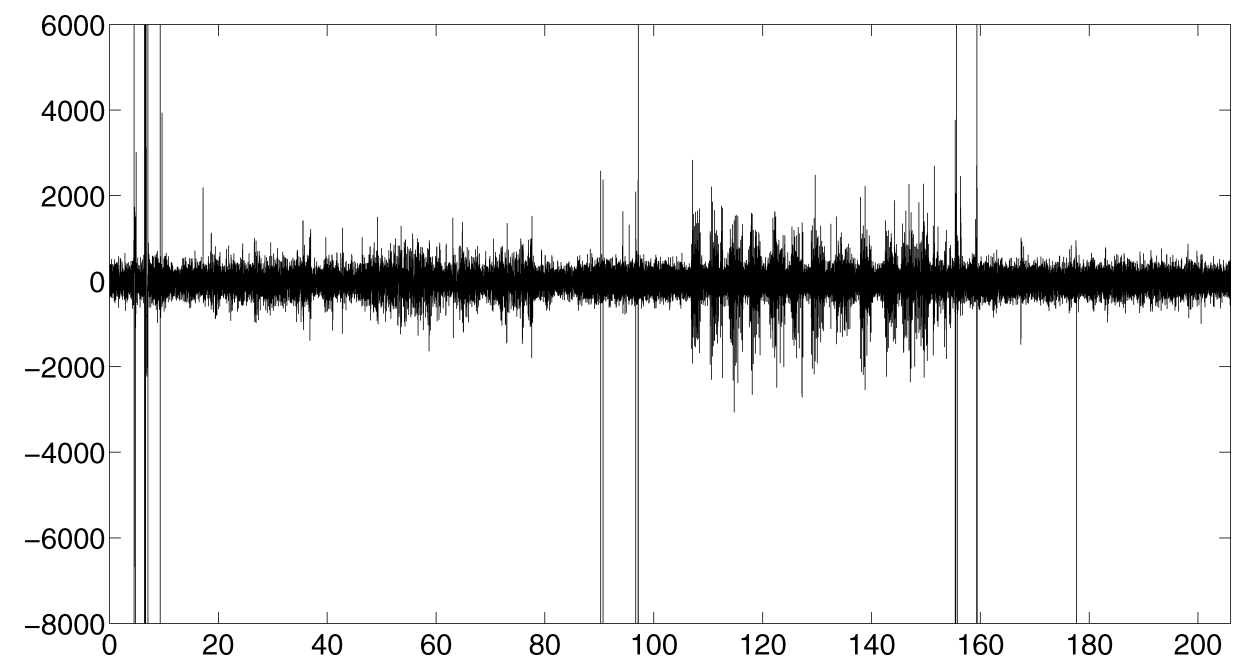

(a)

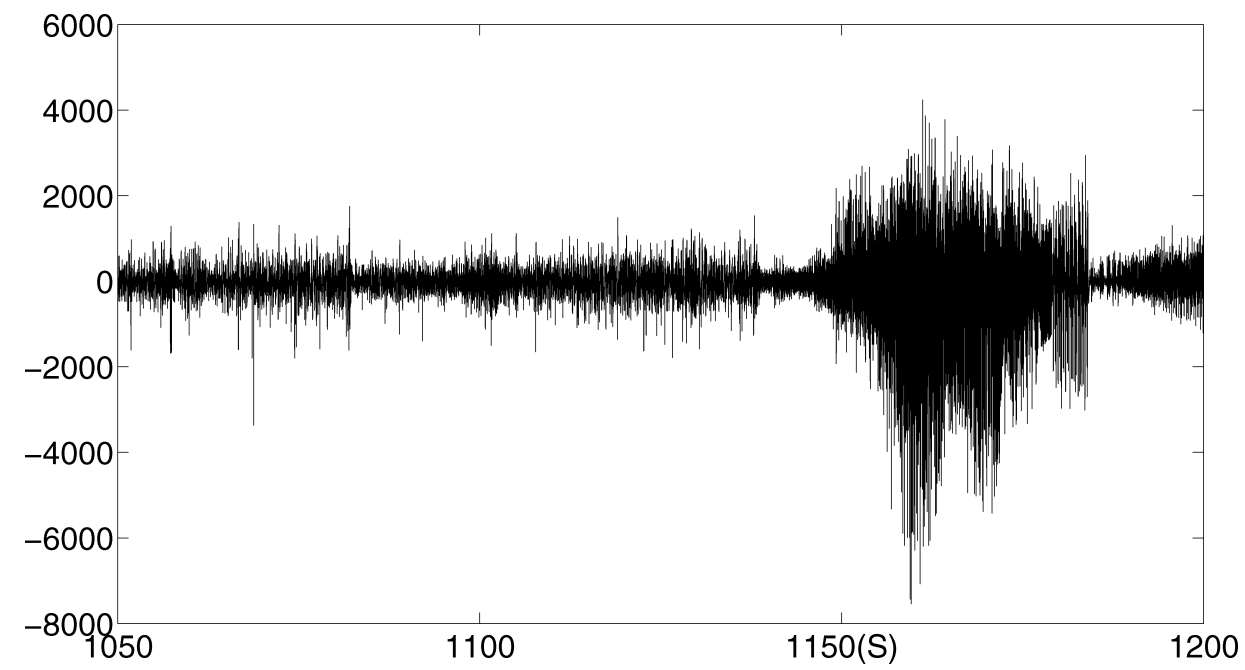

(b)

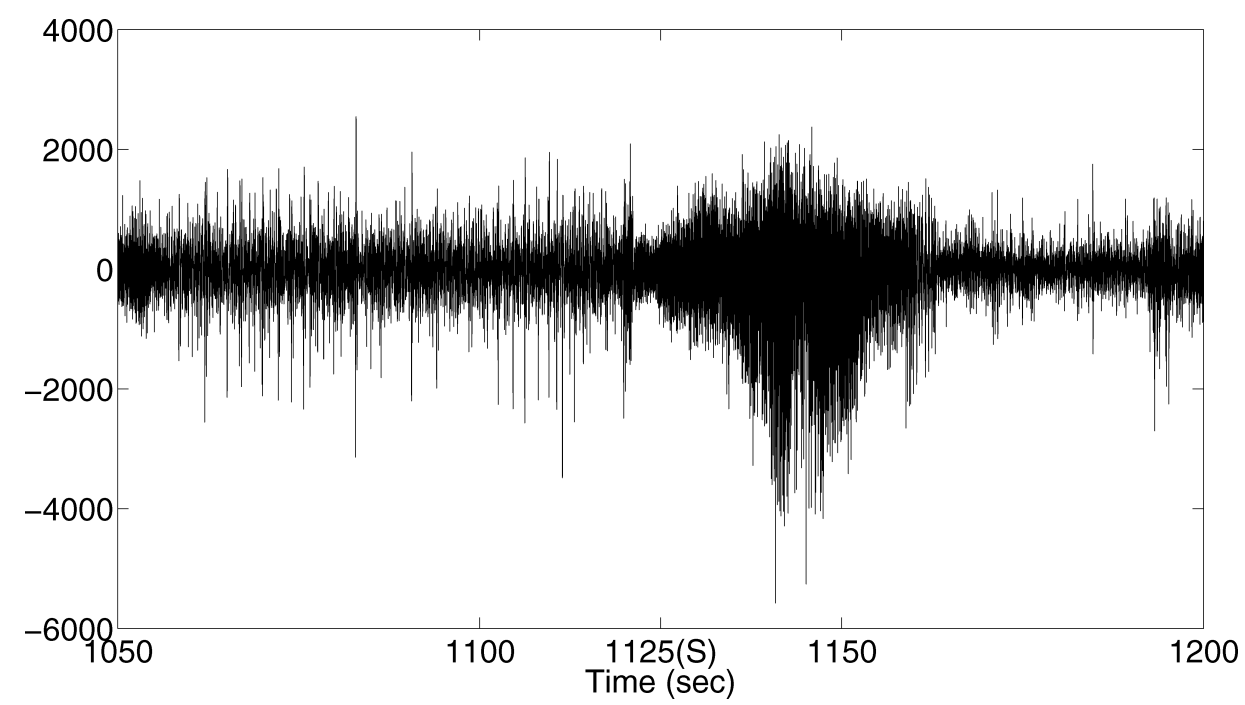

(c)

Fig. 2. A segment of raw EEG trace from channel-1: (a) control, (b) seizure-1 and (c) seizure-2. 
approximately $1120 \mathrm{~s}$ as can be seen from Figs. 2(b) and $2(\mathrm{c})$.

\section{Characterizing Measures of EEG}

The EEG time series, with sampling time $\Delta t=$ $41 \mu \mathrm{s}$, is denoted by $y(i \Delta t)=y_{i}$ where $i=1, \ldots, N$ with $N$ being the sample size. We first compute the first derivative of the EEG time series $x_{i}=y_{i+1}-y_{i}$ for $i=1, \ldots, N-1$ to focus on the short term characteristics of the system [Box \& Jenkins, 1970]. Then we compute the following measures in sliding windows of length 40000 data points $(1.63 \mathrm{~s})$ shifted by 1000 points $(40 \mathrm{~ms})$ : variance $\left(\sigma^{2}\right)$, skewness $(\chi)$, kurtosis $(\kappa)$, relative power in different spectral bandwidths $\left(\delta_{r}, \theta_{r}, \alpha_{r}, \beta_{r}, \gamma_{r}, f_{r}^{1}, f_{r}^{2}, f_{r}^{3}, f_{r}^{4}, f_{r}^{5}\right.$, $f_{r}^{6}, f_{r}^{7}$ and $f_{r}^{8}$ ) (Appendix A.1) and autocorrelation function (ACF) (Appendix A.2). The spectral characterization of the data includes both classical and new bandwidths $\left(f^{1}\right.$ to $\left.f^{8}\right)$ to exploit optimally the high resolution data being investigated (Table 1). The classification of the new spectral bands was done based on the visual inspection of the power spectrum of the data.

Furthermore, we apply nonlinear measures estimated from Recurrence Plots (RPs) [Eckmann et al., 1987] to the EEG data. An RP is a two-dimensional visualization of the trajectory of a dynamical system in phase space. The measures that we use include measures from the Recurrence Quantification Analysis (RQA) [Webber, Jr \& Zbilut, 1994; Zbilut \& Webber Jr, 1992; Zbilut et al., 1998; Marwan \& Kurths, 2005]: Determinism (DET), Mean Diagonal Line Length (L), Laminarity (LAM) and Trapping Time (TT) (Appendix A.3). We also analyze a measure based on Recurrence Time Statistics (RTS) [Gao, 1999; Gao \& Cai, 2000; Ngamga et al., 2007], namely, the Mean Recurrence Time ( $\left.W_{\text {mean }}\right)$ (Appendix A.4).

Table 1. Classification of the spectral bandwidths.

\begin{tabular}{cccc}
\hline Band & $\begin{array}{c}\text { Frequency } \\
\text { Range (Hz) }\end{array}$ & Band & $\begin{array}{c}\text { Frequency } \\
\text { Range (Hz) }\end{array}$ \\
\hline$\delta$ & $0.5-4.0$ & $f^{3}$ & $100-200$ \\
$\theta$ & $4.0-8.0$ & $f^{4}$ & $200-600$ \\
$\alpha$ & $8.0-13.0$ & $f^{5}$ & $600-1000$ \\
$\beta$ & $13.0-30.0$ & $f^{6}$ & $1000-2000$ \\
$\gamma$ & $30.0-48.0$ & $f^{7}$ & $2000-5000$ \\
$f^{1}$ & $48.0-60.0$ & $f^{8}$ & $5000-12000$ \\
$f^{2}$ & $60.0-100.0$ & & \\
\hline
\end{tabular}

\section{Results and Discussion}

In this section, we exemplify the results of our analysis by presenting, comparing and discussing the results from channel-1 of the control and seizure data. Here, it is important to mention that we choose to illustrate our results by considering EEG recordings from channel- 1 for the sake of clarity and consistency.

\subsection{Linear measures}

All the linear measures considered, namely, the moments, the spectral measures and the correlation measures, were able to distinguish the control data (corresponding to a healthy rat) from the seizure data. Furthermore, they indicated the onset of seizures and some pre-ictal events. However, all of them failed to clearly discriminate the pre-ictal warnings from some "random" events which occur in the control data. We illustrate the drawbacks of the linear measures by considering the spectral measures as an example.

Figure 3 shows the spectrogram, i.e. the time evolution of the estimated power spectrum for $200 \mathrm{~s}$ segments of control [Fig. 3(a)] and seizure-1 [Fig. 3(b)] data. It gives an overall picture of the role played by the different bandwidths as defined in Table 1. It is obtained by computing the power spectrum of windowed frames of a given signal.

The artifacts present in the control data are clearly represented by stripe-like structures that extend over the entire frequency range. We observe a decrease of power in the higher frequency range (approximately from 2 to $12 \mathrm{kHz}$ ) in the seizure data, compared to the control data. This observation clearly differentiates the activities of the hippocampus of a healthy and epileptic rat. On the other hand, the onset of the seizure, marked by "S", is associated with an increase of power from 0.5 to $2000 \mathrm{~Hz}$ [Fig. 3(b)]. The spectrogram of the seizure data also indicates seizure like, pre-ictal events, at about $1031 \mathrm{~s}, 1058 \mathrm{~s}, 1100 \mathrm{~s}$ and $1125 \mathrm{~s}$. However, such intermittent peaks, that are associated with an an increase of power in the frequency range from 0.5 to $2000 \mathrm{~Hz}$ were also seen in the control data. E.g. between 100 and $160 \mathrm{~s}$ [Fig. 3(a)].

Since the relative power measures provide a more quantitative definition of the role played by the different frequency bands in different physiological/pathological processes, we proceed to find if it is possible to precisely differentiate between (i) the control and seizure data and (ii) the high 


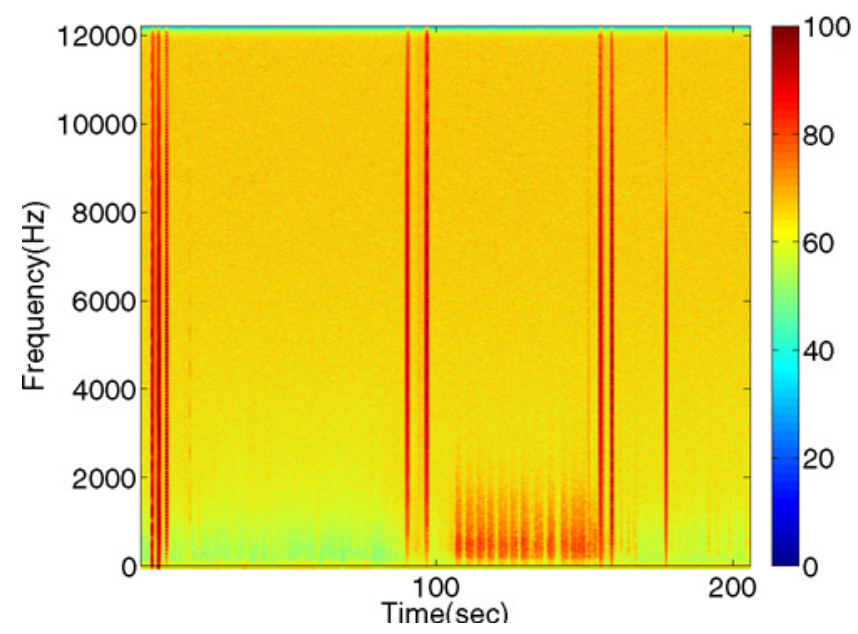

(a)

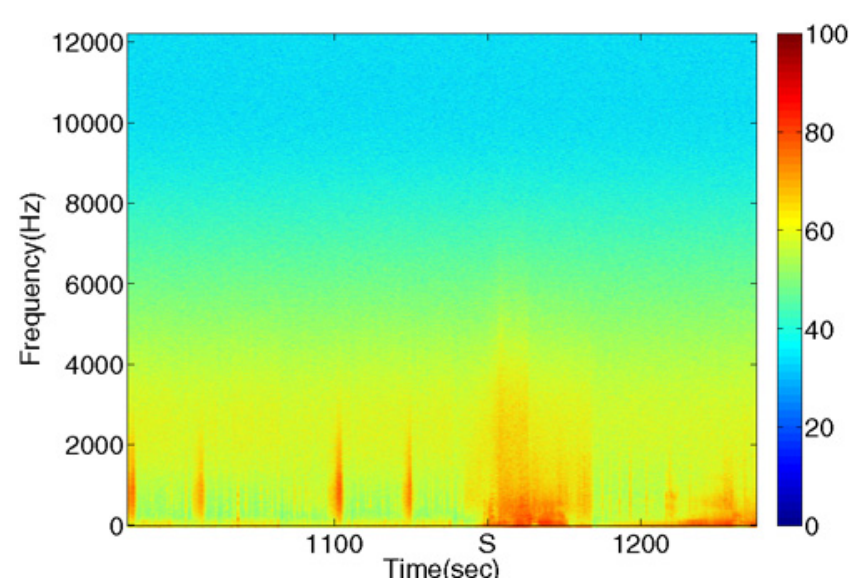

(b)

Fig. 3. Spectrogram of the EEG signal from channel-1 (a) control and (b) seizure-1 (200 s segments). The onset of the seizure is marked by the symbol " $\mathrm{S}$ ".

frequency burst observed in the control and seizure data. The estimated relative power measures discriminated the seizure data from the control data by a decrease in energy in the $5-12 \mathrm{kHz}$ bandwidth $\left(f_{r}^{8}\right.$ (Appendix A.1)), unlike the spectrogram which showed a decrease in the frequency range $2-12 \mathrm{kHz}$. As it can be seen from Fig. $4, f_{r}^{8}$ in the control data fluctuates about $8 \cdot 10^{-1}$ [Fig. 4(a)] and in the seizure data, it fluctuates around $7 \cdot 10^{-2}$. The relative power measures computed for the remaining frequency bands $\left(\delta_{r}, \theta_{r}, \alpha_{r}, \beta_{r}, \gamma_{r}, f_{r}^{1}, f_{r}^{2}, f_{r}^{3}, f_{r}^{4}, f_{r}^{5}\right.$, $f_{r}^{6}$, and $f_{r}^{7}$ (Appendix A.1)) of the seizure data show an increase in energy in the case of seizure data compared to the control data. For example, $f_{r}^{7}$ in the control data fluctuates about $1 \cdot 10^{-1}$ [Fig. 4(a)] and in the seizure data, it fluctuates around $4 \cdot 10^{-1}$ [Fig. 4(b)]. The spectrogram, however, did not show such a clear cut difference between the seizure and the control data in the $0.5-5000 \mathrm{~Hz}$ range.

The onset of the seizure is clearly detected by $f_{r}^{7}$ and $f_{r}^{8}$ (marked by "S" in Fig. 4(b)). Analogous to the spectrogram, we observe strong fluctuations between $900-1150 \mathrm{~s}$ in $f_{r}^{7}$ and $f_{r}^{8}$ just before the onset of the seizure as well as between $400-800 \mathrm{~s}$. It is not clear whether these fluctuations are also pre-ictal warnings, since the magnitude of all the fluctuations are similar to that of events observed during the 100-160 s in the control data. This means that the measures cannot distinguish between preictal warnings and other "random" fluctuations which occur in the control data. Other linear measures investigated are found to have a similar problem.
These results are indicative of the following facts. First, higher frequency oscillations definitely play an important role during the latent period of epileptogenesis as well as during the ictal state. Second, since the EEG recordings from the freely behaving healthy rats also exhibit such intermittent increase of energy in the higher frequency range, their possible role in governing other physiological activities (which are so far not clear) cannot be neglected. Hence, with only the spectral information, it is difficult to characterize the high frequency bursts occurring prior to a seizure as preictal warnings/precursors. In the next section, we show that this problem can be solved by obtaining a dynamical perspective of the data.

\subsection{Nonlinear measures: $R Q A$ and RTS}

Recurrence plots (RPs) and RPs based measures (RQA and RTS) have been shown to be useful for the analysis of a great variety of complex real world systems [Marwan et al., 2007]. In this section, we present the results of recurrence analysis of the EEG recordings. Time profiles of each of the RQA and the RTS measures were computed using sliding windows, as mentioned in Sec. 3. Before calculating the recurrence based measures, we sampled the EEG data to $2.5 \mathrm{kHz}$ to facilitate the computation of the recurrence matrix in each window. Then, the univariate data in each channel was embedded to reconstruct the phase space [Takens, 1981]. The reconstruction was done using an embedding 


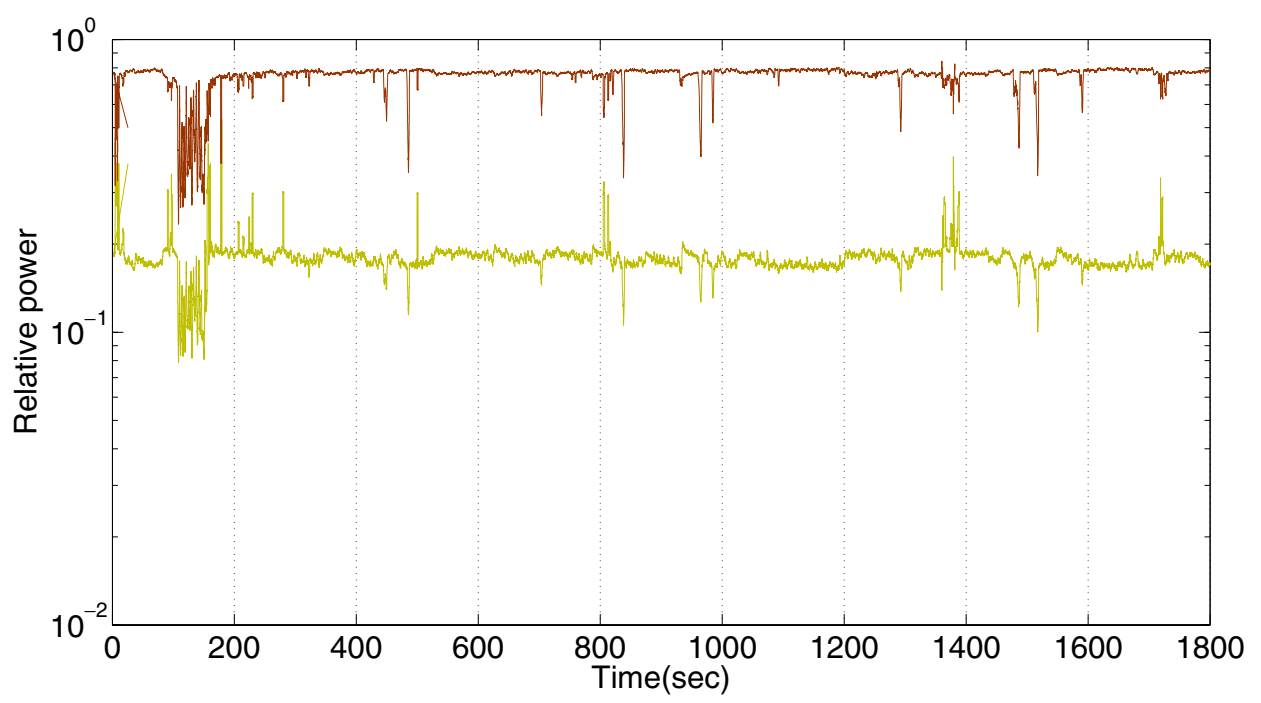

(a)

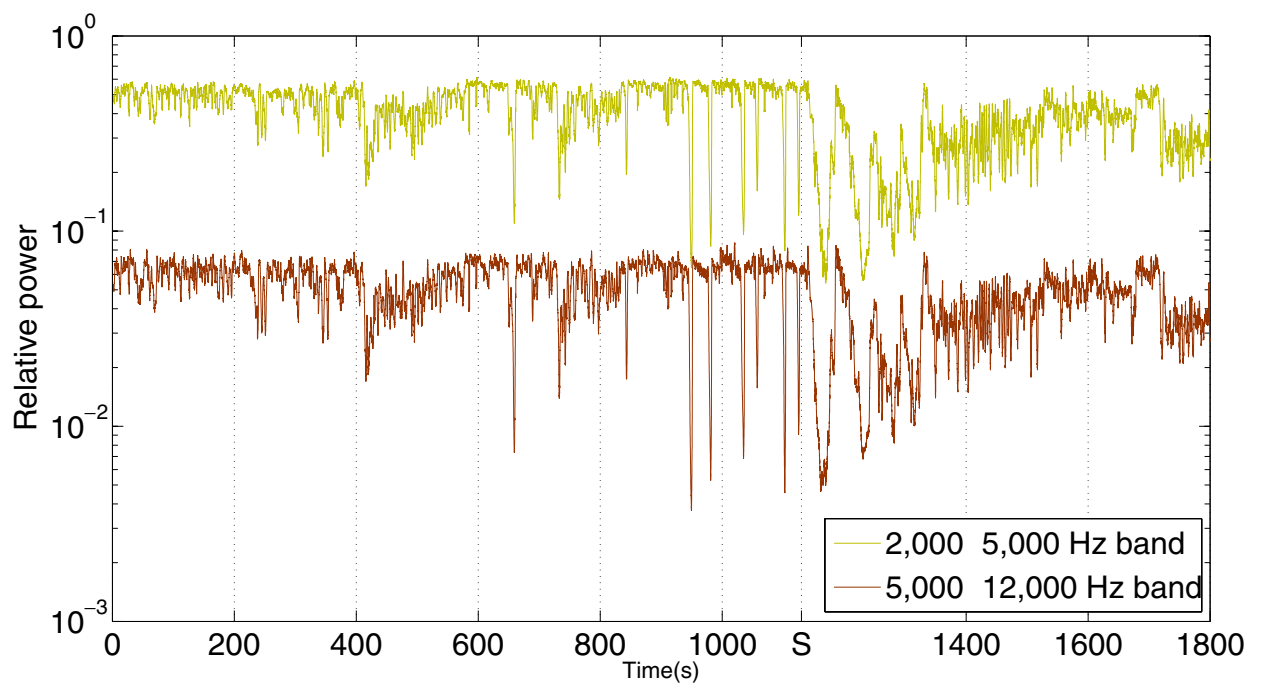

(b)

Fig. 4. Time course of $f_{r}^{7}$ (green line) and $f_{r}^{8}$ (brown line) for (a) control and (b) seizure-1 data for channel-1. The onset of seizure is marked by the symbol " $\mathrm{S}$ ".

dimension of 6 and a time delay of 10 (corresponding to $\approx 4.0 \mathrm{~ms}$ ). A variety of different embedding parameters were tested but the results were found to be qualitatively the same in all the cases studied.

The RPs constructed from each window were made to have the same number of recurrence points, i.e. the threshold $\varepsilon$ defining the size of the neighborhood (see (Appendix A.3)) was chosen so that the recurrence rate [Eq. (A.7)] was the same in all windows $(\mathrm{RR}=0.05)$. This procedure is necessary because if we use the same threshold $\varepsilon$ for all channels and all windows, changes in the amplitude might conceal changes in the dynamics. We know that the onset of seizure is manifested in the EEG recordings as high amplitude activities. Using a fixed threshold $\varepsilon$ for such a signal will be obviously successful only in capturing the nonstationarity in the data. The EEG signals from different channels often have different amplitudes, which require adjusting of $\varepsilon$ for each channel. Other parameters for the RQA were chosen as follows: $l_{\min }=4$ (corresponding to $\approx 1.6 \mathrm{~ms}$ ), $v_{\min }=4$ and Theiler window [Theiler, 1986] of length 1. For other choices of these parameters, the results were again qualitatively the same. 


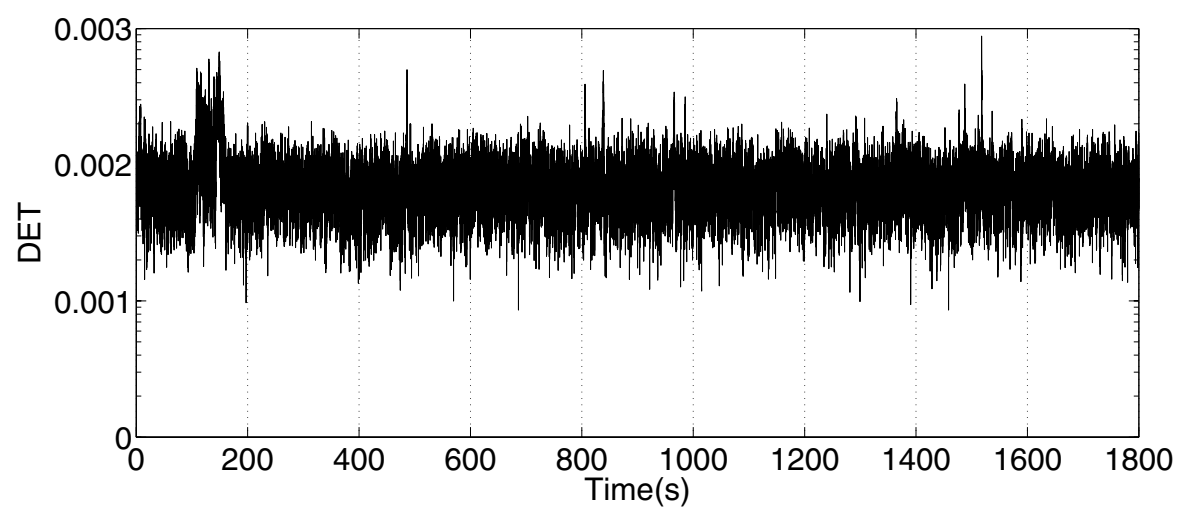

(a)

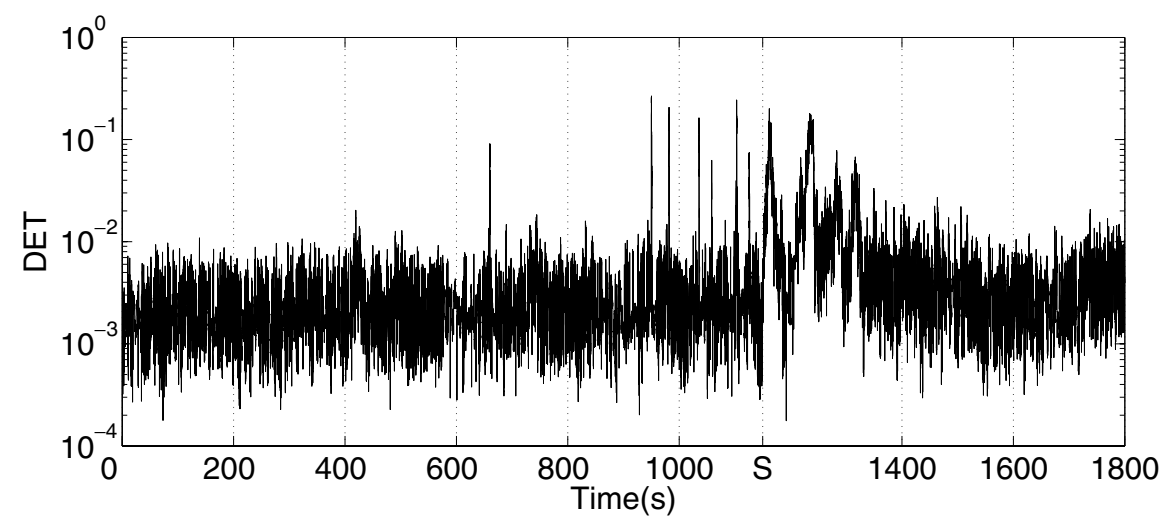

(b)

Fig. 5. Time course of DET for (a) control at channel-1 and (b) seizure-1 data. The onset of seizure is marked by the symbol "S".

The RQA measures allow us to distinguish between control and seizure data. For example, DET [Eq. (A.8)] fluctuates about 0.001 for the control data [Fig. 5(a)] and about 0.003 for the seizure data [Fig. 5(b)]. The RQA measures detect the onset of the seizure clearly (marked as "S" in Fig. 5(b)). Furthermore, the RQA measures fluctuate strongly before the seizure around 900-1150 s [Fig. 5(b)], indicating pre-ictal transitions of the brain. Unlike linear measures, these fluctuations correspond to the pre-ictal warnings and are about two orders of magnitude higher than the "random" fluctuations that occur in the control data [Fig. 5(a)]. Hence, the RQA measures are able to clearly detect the onset of the seizure, as well as recognize the "random" fluctuations.

Also note that DET, which is a measure of predictability of the underlying system, increases at the onset of the seizure and during the warnings. This indicates that the signal becomes more deterministic during the seizure, as well as during the warnings. This result is obtained by fixing the recurrence rate in our analysis, instead of fixing the threshold $\varepsilon$. This enables us to overcome the problem of amplitude fluctuations during the normal, pre-ictal and ictal states and allows us to compare properly the transitions in the brain dynamics. The results are also in accordance with earlier studies which indicate that during the pre-ictal and seizure phases, the brain exhibits more ordered behavior than during inter-ictal phases [Sackellares et al., 2000].

Even though we have presented here only the results for DET (Fig. 5), the results of the other RQA measures also clearly indicate the warnings and the onset of the seizure in almost all channels.

The mean recurrence time $\left(W_{\text {mean }}\right)$ [Eq. (A.12)] does not distinguish between the control and the seizure data [Fig. 6(b)], in contrast to the other measures studied. However, $W_{\text {mean }}$ indicates the onset of the seizures with a sharp increase (marked as "S" in Fig. 6(b)). Moreover, $W_{\text {mean }}$ shows clear 


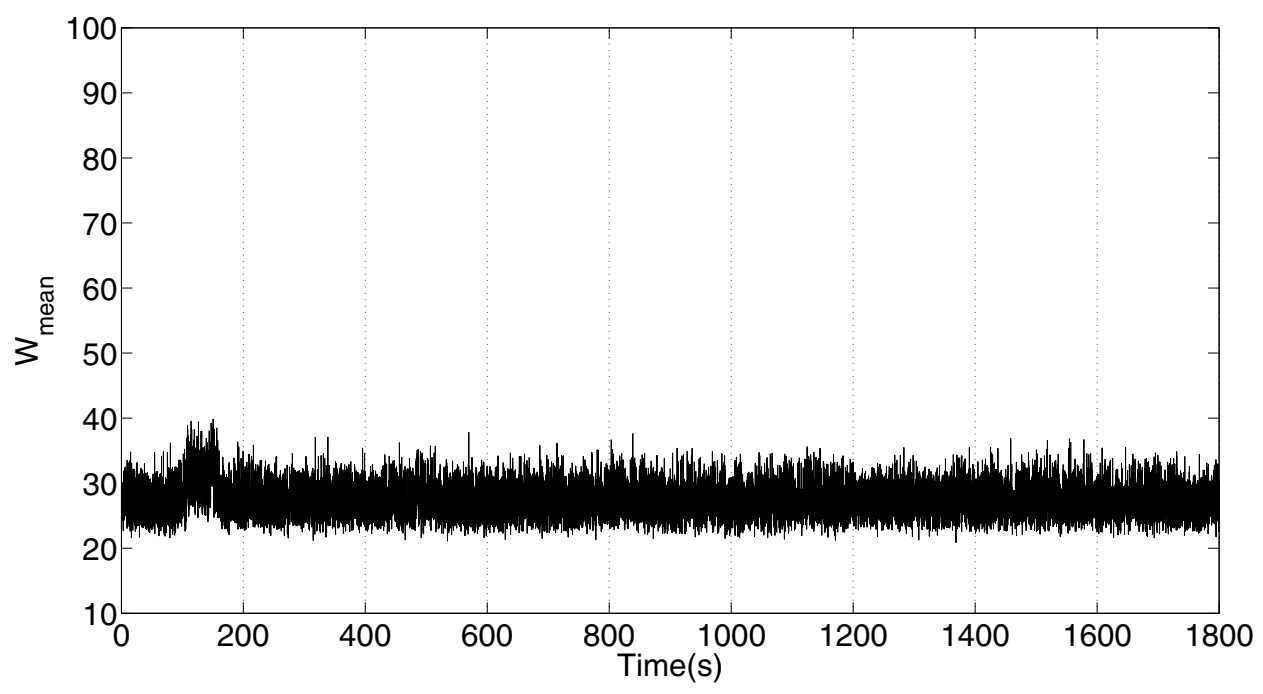

(a)

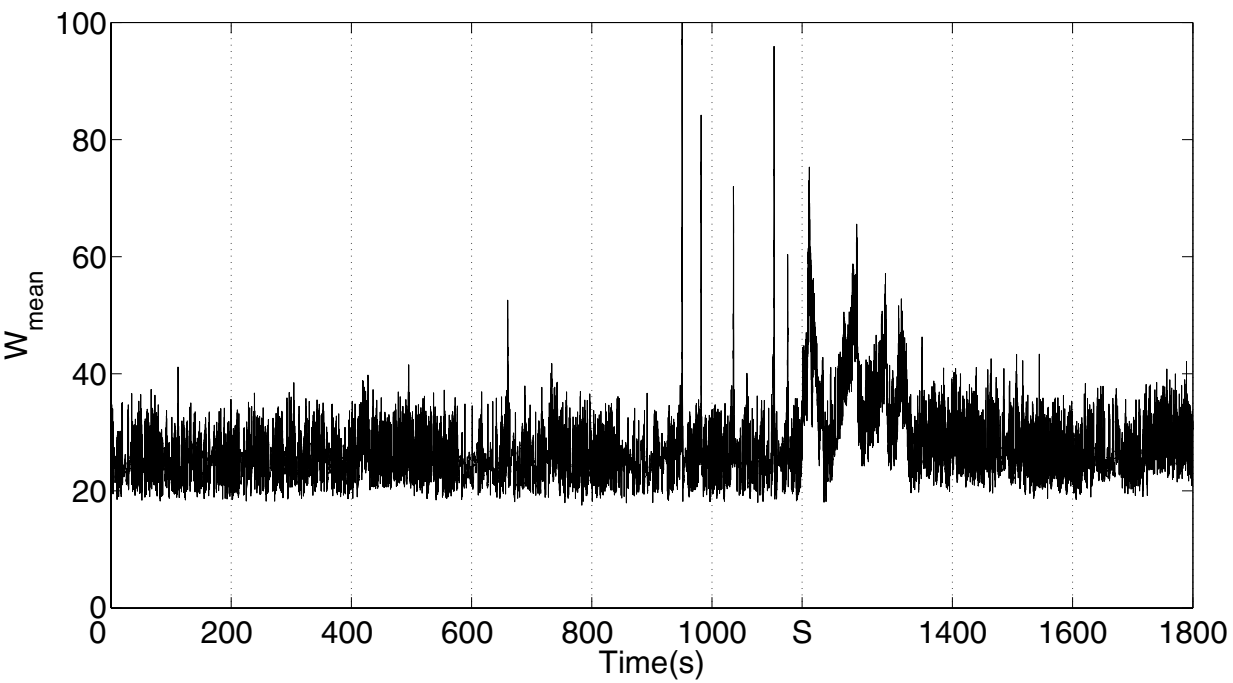

(b)

Fig. 6. Time course of the measure $W_{\text {mean }}$ for (a) control and (b) seizure data from channel-1. The onset of the seizure is marked by the symbol "S".

fluctuations between 900-1150s [Fig. 6(b)] corresponding to the fluctuations found in the RQA measures, which can be interpreted as warnings. However, there were a few channels in which $W_{\text {mean }}$ did not clearly indicate the precursors to the seizures.

\section{Conclusion}

The brain is one of the most interesting and challenging dynamical systems in nature. Significant progress has been made over the last years in understanding the brain at the microscopic level (single neurons and single synapses). However, understanding its dynamics on a macroscopic scale is still a huge task. Studies in this direction could greatly improve our understanding of the basic principles governing information processing in the brain and pathogenesis of neuropsychiatric disorders. Epilepsy is one of the prime candidates to acquire the benefits of this research. Furthermore, understanding the transitions of the brain prior to seizure, is crucial to help prevent life threatening events.

Modern neural interface technology has opened new doors in epileptogenesis research. Recent 
studies support the significance of high frequency oscillations in the pathological and physiological activities. In this paper, we have compared the capability of different univariate linear measures such as moments, spectral band power and autocorrelation function with measures from the Recurrence Quantification Analysis (RQA) and Recurrence Time Statistics (RTS), to analyze very high resolution EEG recordings obtained from a healthy rat (control data) and rats induced with status epilepticus (seizure data).

All linear measures that were investigated distinguish the control and seizure data. Spectral analysis has revealed the role of high frequency oscillations $(>600 \mathrm{~Hz})$ in the highly sampled data. Though all the univariate measures investigated are found to indicate the pre-ictal changes, all of them failed to distinguish between pre-ictal warnings and "random" fluctuations found in the control data. However, recurrence based dynamical measures (RQA and RTS), computed from RPs of EEG recordings, discriminated the high frequency oscillations occurring in the control data from that occurring during pre-ictal/ictal warning periods. They clearly discern between the "random" fluctuations which often occur in the control data, and real warnings which appear prior to a seizure. The results support the hypothesis that the epileptic brain makes intermittent and abrupt transitions into and out of an ictal like state (precursors/warnings) prior to a seizure. The findings also support the fact that pre-ictal and ictal phases are more deterministic than inter-ictal ones. The next steps are to test the statistical validity (sensitivity, specificity, false positives and false negatives, etc.) of our approach by applying it to larger experimental data sets and also to compare the efficiency of our method with the other nonlinear measures that have been proposed for seizure prediction purpose [Mormann et al., 2005; Mormann et al., 2003; Quyen et al., 1999; Lehnertz \& Elger, 1995, 1999].

\section{Acknowledgments}

C. Komalapriya would like to acknowledge the financial support from the Virtual Institute Pole Equator Pole (PEP), NATO projects and MAP A0-99-030 (contract \# 14592) of the Microgravity Application Program/Biotechnology from ESA. C. Komalapriya would also like to thank Dr. S. Talathi for proofreading the manuscript. M. C. Romano would like to acknowledge the
Scottish Universities Life Science Alliance (SULSA) for the financial support. M. Thiel would like to acknowledge the RUCK academic fellowship from EPSRC. This research was supported by the National Institutes of Biomedical Imaging and Bioengineering (NIBIB) through Collaborative Research in Computational Neuroscience (CRCNS) Grant Number R01NS050582-01A1, Bioengineering Research Partnership Grant Number R01EB002089, the Wilder Center of Excellence for Epilepsy Research, and the Children's Miracle Network. We would like to thank the lab technician Angela Haddock who performed all the surgeries for electrode implantation and Dr. Wendy Norman who provided her kind assistance in scanning the EEG/video data to identify the seizures and in creating the control data sets for the statistical analysis.

\section{References}

Box, G. E. P. \& Jenkins, G. M. [1970] Time Series Analysis - Forecasting and Control (Holden Day, San Fransisco).

Bragin, Jr., A., Engel, J., Wilson, C. L., Fried, I. \& Buzsaki, G. [1999a] "High-frequency oscillations in human brain," Hippocampus 9, 137-142.

Bragin, Jr., A., Engel, J., Wilson, C. L., Fried, I. \& Mathern, G. W. [1999b] "Hippocampal and entorhinal cortex high-frequency oscillations (100$500 \mathrm{~Hz}$ ) in human epileptic brain and kainic acidtreated rats with chronic seizures," Epilepsia $\mathbf{4 0}$, 127-137.

Bragin, A., Wilson, C. L., Staba, R. J., Fried, I. \& Engel, Jr., J. [2002a] "Interictal high-frequency oscillations $(80-500 \mathrm{~Hz})$ in human epileptic brain: Entorhinal cortex," Ann Neurol. 52, 407-415.

Bragin, A., Wilson, C. L. \& Engel, Jr., J. [2002b] "Local generations of fast ripples in epileptic brain," $J \mathrm{Neu}$ rosci. 22, 2012-2021.

Bragin, A., Wilson, C. L., Almajano, J., Mody, I. \& Engel, Jr., J. [2004] "High frequency oscillations after status Epilepticus: Epileptogenesis and seizure genesis," Epilepsia 45, 1017-1023.

Buzsaki, G. [1989] "Two-stage model of memory trace formation: A role for noisy brain states," Neuroscience 31, 551-570.

Buzsaki, G. [1996] "The hippocampo-neocortical dialogue," Cereb. Cortex 6, 81-92.

Buzsaki, G. [1998] "Memory consolidation during sleep: A neurophysiological perspective," J. Sleep Res. 7, 17-23.

Buzsaki, G., Horvath, Z., Urioste, R., Hetke, J. \& Wise, K. [1992] "High-frequency network oscillation in hipppocampus," Science 256, 1025-1027. 
Casdagli, M., Iasemidis, L. D., Savit, R. S., Gilmore, R. L., Roper, S. N. \& Sackellars, J. C. [1997] "Nonlinearity in invasive EEG recordings from patients with temporal lobe epilepsy," Electroencephalogr. Clin. Neurophysiol. 79, 98-105.

Clemens, Z., Molle, M., Eross, L., Barsi, P., Halasz, P. \& Born, J. [2007] "Temporal coupling of parahippocampal ripples, sleep spindles and slow oscillations in humans," Brain 130, 2868-2878.

Eckmann, J.-P., Kamphorst, S. O. \& Ruelle, D. [1987] "Recurrence plots of dynamical systems," Europhys. Lett. 5, 973-977.

Firpi, H., Smart, O., Worrell, G., Marsh, E., Dlugos, D. \& Litt, B. [2007] "High frequency oscillations detected in epileptic networks using swarmed neural network feqtures," Ann. Biomed. Engin. 35, 15731584 .

Gao, J. B. [1999] "Recurrence time statistics for chaotic systems and their applications," Phys. Rev. Lett. 83, 3178-3181.

Gao, J. B. \& Cai, J. [2000] "On the structures and quantification of recurrence plots," Phys. Lett. A 270, 75-87.

Hegger, R., Kantz, H. \& Schreiber, T. [1999] "Practical implementation of nonlinear time series methods: The TISEAN package," Chaos 9, 413-435.

Iasemidis, L. D., Shiau, D. S., Pardalos, P. M., Chaovalitwongse, W., Narayanan, K., Prasad, A., Tsakalis, K., Carney, P. R. \& Sackellares, J. C. [2005] "Longterm prospective on-line real-time seizure prediction," Clin. Neurophysiol. 116, 532-544.

Jerger, K. K., Netoff, T. I., Francis, J. T., Sauer, T., Pecora, L., Weinstein, S. L. \& Schiff, S. J. [2001] "Early seizure detection," J. Clin. Neurophysiol. 18, 259-268.

Lai, Y. C, Harrison, M. A. F., Frei, M. G. \& Osorio, I. [2004] "Controlled test for predictive power of Lyapunov exponents: Their inability to predict epileptic seizures," Chaos 14, 630-642.

Lehnertz, K. \& Elger, C. E. [1995] "Spatio-temporal dynamics of the primary epileptogenic area in temporal lobe epilepsy characterized by neuronal complexity loss," Electroencephalogr. Clin. Neurophysiol. 95, 108-117.

Lehnertz, K. \& Elger, C. E. [1999] "Can epileptic seizures be predicted? Evidence from nonlinear time series analysis of brain electrical activity," Phys. Rev. Lett. 80, 5019-5022.

Le Van Quyen, M., Martinerie, J., Baulac, M. \& Varela, F. [1999] "Anticipating epileptic seizures in real time by a non-linear analysis of similarity between EEG recordings," NeroReport 10, 2149-2155.

Li, X., Ouyang, G., Yao, X. \& Guan, X. [2004] "Dynamical characteristics of pre-epileptic seizures in rats with recurrence quantification analysis," Phys. Lett. A 164, 164-171.
Litt, B., Esteller, R., Echauz, J., Alessandro, M. D., Shor, R., Henry, T., Pannell, P., Epstein, C., Bakay, R., Dichter, M. \& Vachtsevanos, G. [2001] "Epileptic seizures may begin hours in advance of clinical onset: A report of five patients," Neuron 30, 51-64.

Marwan, N., Wessel, N., Meyerfeldt, U., Schirdewan, A. \& Kurths, J. [2002] "Recurrence plot based measures of complexity and its application to heart rate variability data," Phys. Rev. E 66, 026702.

Marwan, N. \& Kurths, J. [2005] "Line structures in recurrence plots," Phys. Lett. A 336, 349-357.

Marwan, N., Romano, M. C., Thiel, M. \& Kurth, J. [2007] "Recurrence plots for the analysis of complex systems," Phys. Rep. 438, 237-329.

Mormann, F., Andrzejak, R. G., Krez, T., Reike, C., David, P., Elger, C. E. \& Lehnertz, K. [2003] "Automated detection of a pre-seizure state based on a decrease in synchronization in intracranial EEG recordings from epilepsy patients," Phys. Rev. E. 67, 021912.

Mormann, F., Kreuz, T., Rieke, C., Andrzejak, R. G., Kraskov, A., David, P., Elger, C. E. \& Lehnertz, K. [2005] "On the predictability of epileptic seizures," Clin. Neurophysiol. 116, 569-587.

Mormann, F., Andrzejak, R. G., Elger, C. E. \& Lehnertz, K. [2007] "Seizure prediction: The long and winding road," Brain 130, 314-333.

Ngamga, E. J., Nandi, A., Ramaswamy, R., Romano, M. C., Thiel, M. \& Kurths, J. [2007] "Recurrence analysis of strange nonchaotic dynamics," Phys. Rev. $E \mathbf{7 5}, 0362222$.

Sackellares, J. C., Iasemidis, L. D., Gilmore, R. L. \& Roper, S. N. [2000] Chaos in the Brain (World Scientific, Singapore).

Sanchez, J .C., Mareci, T., Norman, W., Principe, J., Ditto, W. \& Carney, P. R. [2006] "Evolving into epilepsy: Multiscale electrophysiological analysis and imaging in an animal model," Expt. Neurol. 198, $31-47$.

Steuer, R., Ebeling, W., Bengner, T., Dehnicke, C., Hattig, H. \& Meencke, H.-J. [2004] "Entropy and complexity analysis of intracranially recorded EEG," Int. J. Bifurcation and Chaos 14, 815-823.

Takens, F. [1981] Dynamical Systems and Turbulence, in Lecture Notes in Mathematics, Vol. 898 (Springer, Berlin).

Talathi, S. S., Hwang, D. U., Carney, P. R., Ditto, W., Spano, M., Myers, S. \& Winters, J. [2008] "Nonparametric early seizure detection in an animal model of temporal lobe epilepsy," J. Neural Eng. 5, 85-98.

Theiler, J. [1986] "Spurious dimension from correlation algorithms applied to limited time-series data," Phys. Rev. A 34, 2427-2432.

Thomasson, N., Hoeppner, T. J., Webber, Jr., C. L. \& Zbilut, J. P. [2001] "Recurrence quantification in epileptic EEGs," Phys. Lett. A 279, 94-101. 
Webber, Jr., C. L. \& Zbilut, J. P. [1994] "Dynamical assessment of physiological systems and states using recurrence plot strategies," J. Appl. Physiol. 76, 965973.

Worrell, G. A., Parish, L., Cranstoun, S. D., Jonas, R., Blatuch, G. \& Litt, B. [2004] "High-frequeny oscillations and seizure generation in neocortical epilepsy," Brain 127, 1496-1506.

Worrell, G. A., Gardner, A. B., Stead, S. M., Hu, S., Goerss, S., Cascino, G. J., Meyer, F. B., Marsh, R. \& Litt, B. [2008] "High frequency oscillations in human temporal lobe: Simultaneous microwave and clinical macroelectrode recording," Brain 131, 928-937.

Zbilut, J. P. \& Webber, Jr., C. L. [1992] "Embeddings and delays as derived from quantification of recurrence plots," Phys. Lett. A 171, 199-203.

Zbilut, J. P., Giuliani, A. \& Webber, Jr., C. L. [1998] "Detecting deterministic signals in exceptionally noisy environments using cross-recurrence quantification," Phys. Lett. A 246, 122-128.

\section{Appendix A}

\section{A.1. Relative power of spectral bands}

The estimation of the power spectrum [Hegger et al., 1999] of a time series $\left\{x_{i}\right\}_{i=1}^{N}$ is given by the square of the smoothed amplitudes of the Fourier Transform

$$
P_{f}=\left|s_{f}^{2}\right|, \quad f=1, \ldots, \frac{f_{s}}{2},
$$

where $f_{s}=1 / \Delta t$ is the sampling rate, and

$$
s_{f}=\frac{1}{\sqrt{N}} \sum_{i=1}^{N} x_{i} e^{j 2 \pi i f / N},
$$

with $N$ being the length of the considered time series. Based on literature [Mormann et al., 2005; Worrell et al., 2004] and on visual inspection of the spectrogram and power spectrum, we classify the frequency range between $0.5-12000 \mathrm{~Hz}$ into 13 different spectral bands. The relative power contained in the different bands is defined as follows

$$
\begin{aligned}
& \delta_{r}=\frac{1}{P} \sum_{f=0.5 \mathrm{~Hz}}^{4 \mathrm{~Hz}} P_{f} ; \quad \theta_{r}=\frac{1}{P} \sum_{f=4 \mathrm{~Hz}}^{8 \mathrm{~Hz}} P_{f} ; \\
& \alpha_{r}=\frac{1}{P} \sum_{f=8 \mathrm{~Hz}}^{13 \mathrm{~Hz}} P_{f} ; \quad \beta_{r}=\frac{1}{P} \sum_{f=13 \mathrm{~Hz}}^{30 \mathrm{~Hz}} P_{f} ; \\
& \gamma_{r}=\frac{1}{P} \sum_{f=30 \mathrm{~Hz}}^{48 \mathrm{~Hz}} P_{f} ; \quad f_{r}^{1}=\frac{1}{P} \sum_{f=48 \mathrm{~Hz}}^{60 \mathrm{~Hz}} P_{f} ;
\end{aligned}
$$

$$
\begin{aligned}
& f_{r}^{2}=\frac{1}{P} \sum_{f=60 \mathrm{~Hz}}^{100 \mathrm{~Hz}} P_{f} ; \quad f_{r}^{3}=\frac{1}{P} \sum_{f=100 \mathrm{~Hz}}^{200 \mathrm{~Hz}} P_{f} ; \\
& f_{r}^{4}=\frac{1}{P} \sum_{f=200 \mathrm{~Hz}}^{600 \mathrm{~Hz}} P_{f} ; \quad f_{r}^{5}=\frac{1}{P} \sum_{f=600 \mathrm{~Hz}}^{1000 \mathrm{~Hz}} P_{f} ; \\
& f_{r}^{6}=\frac{1}{P} \sum_{f=1000 \mathrm{~Hz}}^{2000 \mathrm{~Hz}} P_{f} ; \quad f_{r}^{7}=\frac{1}{P} \sum_{f=2000 \mathrm{~Hz}}^{5000 \mathrm{~Hz}} P_{f} ; \\
& f_{r}^{8}=\frac{1}{P} \sum_{f=5000 \mathrm{~Hz}}^{12000 \mathrm{~Hz}} P_{f} ;
\end{aligned}
$$

where $P=\sum_{f=0.5 \mathrm{~Hz}}^{12 \mathrm{kHz}} P_{f}$ is the total power in the signal.

\section{A.2. Autocorrelation function}

The autocorrelation function $A(\tau)$ of a time series $\left\{x_{i}\right\}_{i=1}^{N}$ at a lag $\tau$ is defined as

$$
A(\tau)=\frac{\sum_{i=1}^{N-\tau}\left(x_{i}-\bar{x}\right)\left(x_{i+\tau}-\bar{x}\right)}{\sum_{i=1}^{N-\tau}\left(x_{i}-\bar{x}\right)^{2}},
$$

where $\bar{x}$ denotes the mean value. Denoting the first zero crossing of $A(\tau)$ by $\tau_{c}$, we define the following index for a given lag $\tau_{\max }$

$$
A C F=\frac{1}{\tau_{\max }} \sum_{k=1}^{\tau_{\max }}|A(k)|^{1 / k},
$$

which is an estimate for the decay of the envelope of $A(\tau)$ [Lai et al., 2004].

\section{A.3. Recurrence quantification analysis $(R Q A)$}

A Recurrence Plot (RP) is a two-dimensional visualization of the trajectory of a dynamical system in phase space. RPs consist of the graphical representation of the binary matrix

$$
\mathbf{R}_{i, j}=\Theta\left(\varepsilon-\left\|\mathbf{x}_{i}-\mathbf{x}_{j}\right\|\right), \quad i, j=1, \ldots, N
$$

where $\mathbf{x}_{i} \in \mathcal{R}^{d}$ stands for a point in a $d$-dimensional phase space at time $i, \varepsilon$ is a predefined threshold, $\Theta(\cdot)$ is the Heaviside function, and $\|\cdot\|$ is a norm, e.g. the Euclidean or the Maximum norm. The RP 
is then obtained by assigning a black point to the value one and a white point to the value zero.

To quantify the structures found in an RP, a set of measures, called Recurrence Quantification Analysis (RQA), has been proposed [Zbilut \& Webber Jr, 1992; Webber, Jr \& Zbilut, 1994; Zbilut et al., 1998; Marwan \& Kurths, 2005]. We use the following RQA measures for our study:

- Recurrence rate (RR), which is defined as the probability of finding a black point in an RP (note that its definition coincides with that of correlation sum)

$$
\mathrm{RR}=\frac{1}{N^{2}} \sum_{i, j=1}^{N} \Theta\left(\varepsilon-\left\|\mathbf{x}_{i}-\mathbf{x}_{j}\right\|\right) .
$$

- Determinism (DET), which is defined as the percentage of black points forming a diagonal line of at least length $l_{\min }$

$$
\mathrm{DET}=\frac{\sum_{l=l_{\min }}^{N} l P(l)}{\sum_{l=1}^{N} l P(l)},
$$

where $P(l)$ denotes the probability of finding a diagonal line of length $l$ in the RP. It was introduced to quantify the predictability of the system. For a purely periodic system, DET $=1$ and $\mathrm{DET} \rightarrow 0$ for a purely stochastic system.

- Mean diagonal length $(L)$, which is the average length of the diagonal lines found in the RP

$$
L=\frac{\sum_{l=l_{\min }}^{N} l P(l)}{\sum_{l=l_{\min }}^{N} P(l)} .
$$

It is the average time during which two trajectories remain close to each other in phase space.

- Laminarity (LAM), which is defined as the percentage of black points present in a black vertical line of the RP of at least length $l_{\text {min }}$

$$
\mathrm{LAM}=\frac{\sum_{l=l_{\min }}^{N} l P_{v}(l)}{\sum_{l=1}^{N} l P_{v}(l)},
$$

where $P_{v}(l)$ is the probability to find a vertical line of length $l$ in the RP. It quantifies the laminar states present in the given trajectory and is capable of detecting chaos-chaos transitions and intermittency [Marwan et al., 2002].

- Trapping Time (TT), which is defined as the mean black vertical line found in an RP

$$
\mathrm{TT}=\frac{\sum_{l=l_{\min }}^{N} l P_{v}(l)}{\sum_{l=1}^{N} P_{v}(l)},
$$

where $P_{v}(l)$ is the probability to find a vertical white line of length $l$ in the RP. It measures the mean time for which a trajectory will stick to a certain state.

\section{A.4. Recurrence Time Statistics ( $R T S)$}

Recurrence Time Statistics (RTS) are based on the study of the time intervals that a system needs to recur close to a former state. In an RP these time intervals correspond to the white vertical lines. From the distribution of the white vertical lines, we can estimate the mean recurrence time by

$$
W_{\text {mean }}=\frac{\sum_{l=1}^{N} l W(l)}{\sum_{l=1}^{N} W(l)},
$$

where $W(l)$ is the probability to find a vertical white line of length $l$ in the RP. 